\title{
Screening History and Diagnostic Characteristics of Cervical Adenocarcinoma: a Retrospective Study
}

\section{Jing Li}

third affiliated hospital of sun Yat sun University

\section{Minjuan Ye}

Third Affiliated Hospital of Sun Yat-Sen University

\section{Huixia Ye}

Third Affiliated Hospital of Sun Yat-Sen University

Junxian He

Third Affiliated Hospital of Sun Yat-Sen University

Xiaomao Li

Third Affiliated Hospital of Sun Yat-Sen University

Yu Zhang ( $\nabla$ zhangyu6@mail.sysu.edu.cn )

Third Affiliated Hospital of Sun Yat-Sen University

\section{Research article}

Keywords: Cervical cancer, Squamous cell carcinoma, Adenocarcinoma, Screening

Posted Date: August 4th, 2020

DOI: https://doi.org/10.21203/rs.3.rs-43697/v1

License: (c) (1) This work is licensed under a Creative Commons Attribution 4.0 International License. Read Full License 


\section{Abstract}

Background: An increasing trend in incidence of cervical adenocarcinoma has been observed in recent years. This research aims to study the screening history and diagnostic characteristics of cervical adenocarcinoma.

Methods: Cervical cancer patients hospitalized in the Gynecology Department of the Third Affiliated Hospital of Sun Yat-sen University between $1^{\text {st }}$ Jan 2017 and $31^{\text {st }}$ Mar 2020 were included. Cervical screening history investigation and analysis were carried out by medical record data inquiry and case investigation.

Results: (1)The chief complaint of $72.0 \%$ of cervical squamous cell carcinoma was vaginal bleeding. $75.6 \%$ of adenocarcinoma came in for abnormal vaginal discharge, $p<0.001$. (2)The HPV infection rate in adenocarcinoma(74.2\%) was lower than that in squamous cell carcinoma(92.9\%), $p<0.001$. (3)The participation rate of cervical screening before diagnosis of adenocarcinoma(21.2\%) was higher than that of squamous cell carcinoma(2.8\%), $p \bigotimes 0.01$. (4) The proportion of early-stage in adenocarcinoma (46.3\%) was larger than in SCC (28.3\%), $p<0.01$.

Conclusion: Compared to cervical squamous cell carcinoma, HPV infection was found to be less closely associated with adenocarcinoma of uterine cervix. The participation rate and frequency of cervical screening of patients with cervical adenocarcinom were more than squamous cell carcinoma. The screening methods at present may not be sensitive enough for precancerous lesion of adenocarcinoma, but regular and multiple screening are still of great significance for early diagnosis of adenocarcinoma of uterine cervix.

\section{Background}

Cervical cancer is the fourth most frequent cancer and fourth in cancer deaths among women, accounting for $6.6 \%$ of all malignancies in women. In 2018 , about 570,000 women were diagnosed with cervical cancer worldwide, of which 311,000 died ${ }^{[1]}$. China has a large amount of cervical cancer patients with approximately 62,000 new cases and 30,000 deaths in China every year ${ }^{[1]}$.

In the WHO (2014) classification of female reproductive system tumors ${ }^{[2]}$, cervical cancer is divided into squamous cell carcinoma (SCC), adenocarcinoma (AC), adenosquamous cell carcinoma (ASC), groundglass cell carcinoma, adenoid basal cell carcinoma, undifferentiated carcinoma, neuroendocrine tumor, etc. Among all pathological types of cervical cancer, SCC comes first in incidence rate, AC comes second, and other pathological types of cervical cancer follow. Cervical intraepithelial lesion is a premalignant condition that can potentially progress to cervical cancer. Early detection of cervical intraepithelial lesions and timely intervention measures can effectively reduce the incidence of cervical malignancy ${ }^{[3,4]}$. Since the 1980s, HPV infection has been internationally recognized as an important pathogenic factor of cervical cancer ${ }^{[5,6]}$. With prevention methods of human papillomavirus (HPV) vaccination and cervical 
screening having significantly reduced the incidence of cervical cancer in the past few decades ${ }^{[8]}$, cervical cancer is largely preventable ${ }^{[7]}$. However, relevant studies in recent years have shown that the decrease of cervical cancer incidence is mainly attributed to the reduction of SCC. The number of AC incidences, on the other hand, has shown an obvious upward trend ${ }^{\left[{ }^{[-12]}\right.}$. From the 1970 s to the 2010 s, the proportion of SCC incidence in cervical cancer diseases has decreased from $95 \%$ to $67-83 \%$ while that of AC has increased from $5 \%$ to $10-25 \%{ }^{[12-14]}$. Thus, the increase of $A C$ is not only reflected in its proportion in cervical cancer diseases but also in the absolute number of incidences ${ }^{[13-14]}$. So, what is the underlying reason for the increasing incidence of $\mathrm{AC}$ ? And are there any differences between the screening history and diagnostic characteristics of AC and SCC? In this study, we aim to investigate the screening history (characteristics of cervical screening participation) and diagnostic characteristics of different histological types of cervical carcinoma.

\section{Methods}

\section{Basic Information Collection}

We collected data on all cervical cancer cases diagnosed in the Third Affiliated Hospital Of Sun Yat-sen University during 2017-2020. All the selected cases were confirmed to be cervical cancer by histopathological examination, and patients with different histological types of cervical cancer were grouped according to the the 2014 World Health Organization (WHO) classification of tumours of female reproductive organs ${ }^{[2]}$. According to the standards, patients with different histological types were divided into three groups: (1)Histopathological diagnosis of cervical squamous cell carcinoma group(SCC): Keratinized squamous cell carcinoma; Non-keratinized squamous cell carcinoma; Papillary squamous cell carcinoma; Basal cell-like squamous cell carcinoma; Condyloma squamous cell carcinoma; Verrucous squamous cell carcinoma; Squamous transitional cell carcinoma.(2)Histopathological diagnosis of cervical adenocarcinoma group(AC):Cervical adenocarcinoma, common type; mucinous adenocarcinoma, non-specific types(including gastric mucinous adenocarcinoma, intestinal mucinous adenocarcinoma and sigma-ring cell mucinous adenocarcinoma); Villous associated carcinoma; Endometrioid carcinoma; Clear cell carcinoma; Serous cancer; Middle renal tubular adenocarcinoma; Adenocarcinoma mixed with neuroendocrine carcinoma. (3)Pathological diagnosis of other types of cervical cancer (Other): Adenosquamous carcinoma; Ground-glass cell carcinoma; Adenoid base cell carcinoma; Adenoid cystic carcinoma; Undifferentiated cancer; Neuroendocrine tumor; Others.

History collection were carried out by electronic medical record data inquiry, telephone follow-up and case investigation. Data collection mainly included basic information (month/year of birth, month/year of diagnosis, age at menarche, menopausal age, reproductive history, etc), chief complaint, the history of participation in cervical cancer screening before diagnosis, the results of HPV test and cytology, histopathological results, and cancer staging. Patients who underwent surgery were staged according to the pathological stage of cancer at the time of surgery. Those who didn't receive surgery were staged by 
clinical stages. We defined stage I as Early-Stage Cervical Cancer and stage II -IV as advanced cervical cancer.

\section{Screening History}

In 2018, consensus guidelines were issued for cervical screening in US populations ${ }^{[16-17]}$, recommending screening begin at age 21yrs; 3-yearly cytology for women aged 21-29yrs, and either 3-yearly cytology, 5yearly primary HPV testing or 5-yearly co-testing(co-occurring HPV and cytology testing) for women 30$65 y$ rs. Most women aged $>65 y$ rs can cease cervical screening ${ }^{[16-17]}$. Based on the patient's participation in cervical cancer screening between 6 months and 5 years prior to diagnosis( since cancers histologically diagnosed within 6 months of an abnormal screening result were almost certainly present at the time of the diagnosis) ${ }^{[18]}$, we grouped the patients into four groups, including "Never screening, Irregular screening, Regular screening, and Frequent screening"(Figure 1). Females who had never attended the screening program before diagnosis were grouped into "Never screening"; females who had attended more than the frequency of recommendation were grouped into "Frequent screening"; females who had attended the screening as the frequency of recommendation were grouped into "Regular screening"; females who had attended screening before but hadn't participated in the most recent screening cycle were grouped into "Irregular screening".(A screening cycle is the recommended interval for cervical screening). Cervical cancer screening participation rate is defined as the ratio of the number of patients participating in screening to the number of all patients.

\section{Statistical Analysis}

We presented the clinical characteristics, the features of HPV infection and the screening history in the past five years before diagnosis of cancer among all cervical cancer cases. Quantitative data were described by Mean (Standard deviation), qualitative data was described by adoption rate or percentage. Comparison among different groups of quantitative data was conducted by T-test or ANOVA, and the chisquare test was used for the comparison of quantitative data. Analyses were stratified by histopathological type. Data manipulation and statistical analyses were conducted in SPSS 25.0.

This study was approved by the medical ethics committee of the clinical research center of the Third Affiliated Hospital of Sun Yat-sen University.

\section{Results}

With the exclusion of those that had no histopathological results, a total of 291 women were diagnosed with cervical cancer in The Third Affiliated Hospital of Sun Yat-sen University between 1st January 2017 and 31st March 2020. Of these 291 cases, $81.1 \%$ were squamous, $15.5 \%$ adenocarcinoma and $3.4 \%$ other histopathological types. The latter group was excluded from further analysis.

The characteristics of different histopathological types of cervical carcinoma were compared in Table 1. The mean age of SCC, AC and other histopathological types of cervical cancer were $50.31 \pm 10.45$ years, 
$46.44 \pm 10.48$ years and $56.50 \pm 9.57$ years. The menarche age of other histopathological types of cervical cancer $(16.67 \pm 6.51)$ was higher than that of SCC and $A C(p \otimes 0.001)$, while there was no significant difference between $\operatorname{SCC}(13.64 \pm 1.33)$ and $A C(13.57 \pm 0.85)(p \otimes 0.05)$. The majority of the patients sought medical attention for vaginal bleeding and abnormal vaginal discharge. The chief complaint of $72.0 \%$ of SCC was vaginal bleeding. $75.6 \%$ of AC came in for abnormal vaginal discharge. The HPV infection rate in AC was $72.4 \%$, and $92.9 \%$ in SCC which was significantly higher than $A C(p<$ $0.001)$. The proportion of cancer stages differed for SCC and AC significantly $(p<0.05)$. The percentages of stage I to IV in SCC were $28.3 \%, 59.9 \%, 9.4 \%, 2.4 \%$ respectively, and in AC were $46.3 \%, 43.9 \%, 4.9 \%$, $4.9 \%$ respectively. Compared to SCC, the proportion of early-stage was significantly larger in AC (19/41, $46.3 \%)$ than in SCC $(60 / 212,28.3 \%)(p<0.01)$. From the results so far, we didn't find any significant differences of histopathological types of cervical carcinoma in age, menopausal age, marital and reproductive history (including number of pregnancies, abortions, natural births and the age of first birth or first abortion) and educational levels ( $p \otimes 0.05)$.

In further analysis by cancer stage(Table 2), the reasons for hospitalizing behavior between SCC and AC, AC in different cancer stages, SCC in different cancer stages, SCC and AC in same cancer stage were compared. The results show that the percentage of patients with $\mathrm{AC}$ coming for abnormal cancer screening results was significantly higher than SCC (AC: $26.8 \%$, SCC: $11.3 \%, p<0.01$ ). But there were no significant differences between AC in different cancer stages (early stage AC:31.6\%, advanced AC: $9.1 \%$, $p=0.070$ ), SCC in different cancer stages (early stage SCC: $18.3 \%$, advanced SCC: $8.6 \%, p=0.054$ ), and SCC or AC in same cancer stage( $p \bigotimes 0.05)$.

Among HPV-positive cervical cancer cases, as shown in Table 3, the infection rate of HPV 16 is the highest both in SCC and AC, while the proportion of HPV-16 infection in SCC was $68.7 \%$, significantly higher than AC $(56.5 \%, p<0.05)$. The infection rate of HPV-18 in SCC was $12.2 \%$, significantly lower than AC $(21.7 \%, p<0.05)$. The infection rate of HPV $52,58,33,31$ in SCC was $6.1 \%, 7.6 \%, 4.6 \%$ and $2.3 \%$ respectively, and the infection rate of HPV 52,31 in AC was $4.3 \%$ and $8.7 \%$. The multiple infection rate in $\operatorname{AC}(16.8 \%, 22 / 131)$ was significantly lower than SCC $(4.3 \%, 1 / 23)(p \otimes 0.05)$.

Among 214 cases with complete information about screening history and histopathological types(Fig. 2), this study found the participation rate of $A C$ in cervical cancer screening(21.2\%) was significantly higher than $\operatorname{SCC}(2.8 \%)(p \otimes 0.01)$. The percentage of Frequent screening and Regular Screening in $\mathrm{AC}(18.2 \%)$ was also higher than $\operatorname{SCC}(1.1 \%)(p \otimes 0.01)$. For early stage cancer, the percentage of Frequent screening and Regular Screening was significantly higher in $\mathrm{AC}(28.6 \%)$ than in $\operatorname{SCC}(3.9 \%)(p \llbracket 0.05)$, as was for advanced stages where the percentage for $A C$ was $10.5 \%$ and $0 \%$ for SCC ( $p \otimes 0.05)$. There was no significant difference between different cancer stages of $A C$ in frequency of screening participation ( $p \bigotimes 0.05)$, nor was there in different cancer stages of $\operatorname{SCC}(p \otimes 0.05)$.

\section{Discussion}


HPV16, 18 are still the most common types in HPV positive cases. HPV infection rate is higher in SCC patients than in AC, indicating a closer relationship between SCC and HPV infection, this conclusion is also supported in previous studies ${ }^{[19-21]}$. Recently, an international group of gynaecological pathologists endeavoured to establish the International Endocervical Adenocarcinoma Criteria and Classification (IECC), a scheme supported by aetiology and based on the presence or absence of HPV infection related morphological features ${ }^{[22]}$. Some studies then found that worse disease-free survival (DFS) and diseasespecific survival (DSS) correlated with non-HPV related adenocarcinoma (NHPVA) group ${ }^{[23]}$, which alert clinicians to take great care of AC with HPV negative results.

Regardless of histopathological types, most patients in this study came in with symptoms. Vaginal bleeding and abnormal vaginal discharge were the main clinical manifestations. Vaginal discharge was much more common in AC than in SCC, reminding clinicians to be alert of the possibility of $A C$ when patients come in for vaginal discharge ${ }^{[24]}$. Interestingly, the percentage of patients that came in for abnormal cervical screening results in AC was significantly higher than in SCC. Figure 2 further compares the frequency of cervical screening before diagnosis in different histopathological types in different stages. Results show that whether irregular screening, regular screening or frequent screening, participation rates of cervical screening before diagnosis of AC were all significantly higher than that of SCC. The difference in screening frequency between early stage AC and advanced AC is not significant. Combined with the characteristics of chief complaints of different histopathological types from Table 1 and Table 2, we suspect that the efficacy of the present cervical cancer screening methods is lacking in detection of AC, regardless of the cancer stage. Patients with squamous intraepithelial lesion could be detected promptly by cervical cancer screening and intervention could take place early on to prevent cancer development. As for patients with glandular intraepithelial lesion, a large proportion of AC patients were found to have had attended screening several times before but still could not be diagnosed timely. Recent study also found that being screened with normal results in two screening cycles was associated with $89 \%$ risk reduction for squamous cell cancer, compared to women unscreened, but only $60 \%$ reduction for adenocarcinoma. ${ }^{[22]}$

Why does the screening method at present show different efficacy in different histopathological types? The reasons may be as follows: Firstly, the precancerous lesions of different histopathological types of cervical cancer have different primary sites. Glandular intraepithelial lesion (GIN), the precancerous lesion of $\mathrm{AC}$, occurs in the depth of the cervical scale-column junction, where the cancer cells are arranged in an interlaced pattern. Thus, fewer abnormal adenocarcinoma cells may be in the cervical exfoliated cells, resulting in difficulty in obtaining abnormal $\mathrm{AC}$ cells by cervical brush. Therefore, cytological screening has poorer detection on $\mathrm{AC}^{[26,27]}$. In contrast, cervical squamous cell carcinoma is easier to obtain abnormal exfoliated cells, which can be detected by cytological screening of precancerous lesions, so as to effectively prevent squamous cell carcinoma. Secondly, the heterogeneous changes in the nucleus in cervical adenocarcinoma, especially in early stage adenocarcinoma, is not as significant as that in squamous cell carcinoma. In particular, highly differentiated mucinous adenocarcinoma often leads to neglect of film reading ${ }^{[28]}$. Thirdly, the HPV infection rate varies for different histopathological cervical 
cancers. In this study, we found the HPV infection rate higher in SCC than in AC (Table 1) ${ }^{[21]}$. In spite of the higher HPV infection rate, some research found that SCC also has a higher HPV viral load than AC [21]. Since the low viral infection rate in AC and the viral DNA integration in host cells, HPV virus is not easy to be detected in AC specimens ${ }^{[29]}$. Hence, to improve the detection accuracy of cervical cancer, co-testing (HPV and cytology testing) in screening program is an optimal choice. Moreover, further study of etiology and pathological development of cervical adenocarcinoma, as well as more efficient cervical screening methods, such as improved techniques of HPV DNA testing to confirm the presence or absence of HPV infection, reformed methods of brushing abnormal AC cells, and artificial intelligent cervical screening program are needed.

In our study, the proportions of the stages of which SCC and AC were presented in were quite different. The percentage of stage I in SCC were lower than that in AC, but the percentage of stage II-IV in SCC were significantly higher than AC. We suspect that may be associated with the frequency of cervical screening. Figure 2 shows that the percentage of SCC that had never been screened was $97.2 \%$, which is significantly higher than that of AC (78.8\%). Patients who had never attended screening before always sought medical attention because of symptoms, by which time the disease had always progressed to an advanced stage. And that may explain why the percentage of SCC in advanced stage is higher than early stage SCC. Table 2 shows that the percentage of AC patients who sought treatment for abnormal screening results in early stage was $31.6 \%$, and $9.1 \%$ in advanced stage $(p=0.07)$, which may suggest that while screening methods at present may not be sensitive enough for adenocarcinoma, regular or multiple screening is still of great significance for early detection. The reason why there was no significant difference may be related to the sample size. However, more evidence and lager studies are still needed to investigate whether different pathological types of cervical cancer have different cancer staging.

\section{Conclusion}

In conclusion, we found many consistent evidences for a relationship between the participation of cervical screening and different histopathological types of cervical cancer. Compared to SCC, HPV infection was found to be less closely associated with AC. The participation rate and frequency of cervical screening of patients with AC was more than SCC. The screening methods at present may not be sensitive enough for precancerous lesion of adenocarcinoma, and regular and multiple screening are still of great significance for early detection. In order to improve the detection accuracy of cervical cancer, cotesting (HPV and cytology testing) in screening program is an optimal choice. Further study of etiology and pathological development of cervical adenocarcinoma as well as more efficient cervical screening methods like reformed techniques of HPV DNA testing and cytological examination, artificial intelligent cervical screening program are needed to be studied to decrease the incidence of cervical adenocarcinoma.

\section{Abbreviations}

HPV: human papilloma virus 
SCC: cervical squamous cell carcinoma

AC:cervical adenocarcinoma

IECC: International Endocervical Adenocarcinoma Criteria and Classification

SIL: squamous intraepithelial lesion

GIN: glandular intraepithelial neoplasia

\section{Declarations}

\section{Ethics approval and consent to participate}

This study was approved by the medical ethics committee of the clinical research center of Third Affiliated Hospital of Sun Yat-sen University. Due to anonymous analyses of the data, the committee concluded that informed consent was not required. The management and publication of patients' information in this study was strictly in accordance with the Declaration of Helsinki, including the confidentiality and anonymity.

\section{Consent for publication}

Not applicable.

\section{Availability of data and materials}

The data that support the findings of this study are available from the corresponding author upon reasonable request.

\section{Competing interests}

None declared.

\section{Funding}

No dedicated funding source was involved in this project.

\section{Authors' contributions}

$\mathrm{J} L, M J \mathrm{Y}, \mathrm{HY}$ and $\mathrm{J} \mathrm{H}$ collected and analyzed patients data. $\mathrm{J} \mathrm{L}$ and $\mathrm{MJ} \mathrm{Y}$ were major contributors in writing this paper. All authors reviewed and edited the manuscript. All authors read and approved the manuscript.

\section{Acknowledgements}

Not applicable. 


\section{References}

1. Bray Freddie., Ferlay Jacques., Soerjomataram Isabelle., Siegel Rebecca L., Torre Lindsey A., Jemal Ahmedin.(2018). Global cancer statistics 2018: GLOBOCAN estimates of incidence and mortality worldwide for 36 cancers in 185 countries. CA Cancer J Clin, 68(6), 394-424.

2. Kurman R, Carcangiu M, Herrington C. WHO Classification of Tumours of Female Reproductive Organs. 4th edn. Lyon: WHO, 2014.

3. Ronco Guglielmo., Dillner Joakim., Elfström K Miriam., Tunesi Sara., Snijders Peter J F., Arbyn Marc., Kitchener Henry., Segnan Nereo., Gilham Clare., Giorgi-Rossi Paolo., Berkhof Johannes., Peto Julian., Meijer Chris J L M., International HPV screening working group.(2014). Efficacy of HPV-based screening for prevention of invasive cervical cancer: follow-up of four European randomised controlled trials. Lancet, 383(9916), 524-32.

4. Qiao YL, Zhao YQ.(2015). Epidemiology and prevention of cervical cancer. Chinese Journal of Obstetrics \& Gynecology and Pediatrics(Electronic Edition), 11(02):1-6.

5. Gissmann L., deVilliers E M., zur Hausen H.(1982). Analysis of human genital warts (condylomata acuminata) and other genital tumors for human papillomavirus type 6 DNA. Int. J. Cancer, 29(2), $143-6$.

6. Dochez Carine., Bogers Johannes J., Verhelst Rita., Rees Helen.(2014). HPV vaccines to prevent cervical cancer and genital warts: an update. Vaccine, 32(14), 1595-601.

7. Landy Rebecca., Sasieni Peter D., Mathews Christopher., Wiggins Charles L., Robertson Michael., McDonald Yolanda J., Goldberg Daniel W., Scarinci Isabel C., Cuzick Jack., Wheeler Cosette M., New Mexico HPV Pap Registry Steering Committee.(2020). Impact of screening on cervical cancer incidence: A population-based case-control study in the United States. Int. J. Cancer, 147(3), 887-896.

8. Adegoke Olusola., Kulasingam Shalini., Virnig Beth.(2012). Cervical cancer trends in the United States: a 35-year population-based analysis. J Womens Health (Larchmt), 21(10), 1031-7.

9. Mathew Aleyamma., George Preethi Sara.(2009). Trends in incidence and mortality rates of squamous cell carcinoma and adenocarcinoma of cervix-worldwide. Asian Pac. J. Cancer Prev., 10(4), 645-50.

10. Ojamaa Kristiina., Innos Kaire., Baburin Aleksei., Everaus Hele., Veerus Piret.(2018). Trends in cervical cancer incidence and survival in Estonia from 1995 to 2014. BMC Cancer, 18(1), 1075.

11. Smith H O., Tiffany M F., Qualls C R., Key C R.(2000). The rising incidence of adenocarcinoma relative to squamous cell carcinoma of the uterine cervix in the United States-a 24-year population-based study. Gynecol. Oncol., 78(2), 97-105.

12. Bucchi Lauro., Baldacchini Flavia., Mancini Silvia., Ravaioli Alessandra., Giuliani Orietta., Vattiato Rosa., Falcini Fabio., Giorgi Rossi Paolo., Campari Cinzia., Canuti Debora., Di Felice Enza., Sassoli de Bianchi Priscilla., Ferretti Stefano., Bertozzi Nicoletta., Biggeri Annibale., Emilia-Romagna Region Workgroup for Cervical Screening Evaluation.(2019). Estimating the impact of an organised 
screening programme on cervical cancer incidence: A 26-year study from northern Italy. Int. J. Cancer, 144(5), 1017-1026.

13. Wang Sophia S., Sherman Mark E., Hildesheim Allan., Lacey James V., Devesa Susan.(2004). Cervical adenocarcinoma and squamous cell carcinoma incidence trends among white women and black women in the United States for 1976-2000. Cancer, 100(5), 1035-44.

14. Vizcaino A P., Moreno V., Bosch F X., Muñoz N., Barros-Dios X M., Parkin D M.(1998). International trends in the incidence of cervical cancer: I. Adenocarcinoma and adenosquamous cell carcinomas. Int. J. Cancer, 75(4), 536-45.

15. Taylor Jennifer., McCluggage W Glenn.(2015). Ovarian seromucinous carcinoma: report of a series of a newly categorized and uncommon neoplasm. Am. J. Surg. Pathol., 39(7), 983-92.

16. Saslow Debbie., Solomon Diane., Lawson Herschel W., Killackey Maureen., Kulasingam Shalini L., Cain Joanna., Garcia Francisco A R., Moriarty Ann T., Waxman Alan G., Wilbur David C., Wentzensen Nicolas., Downs Levi S., Spitzer Mark., Moscicki Anna-Barbara., Franco Eduardo L., Stoler Mark H., Schiffman Mark., Castle Philip E., Myers Evan R., ACS-ASCCP-ASCP Cervical Cancer Guideline Committee.(2012). American Cancer Society, American Society for Colposcopy and Cervical Pathology, and American Society for Clinical Pathology screening guidelines for the prevention and early detection of cervical cancer. CA Cancer J Clin, 62(3), 147-72.

17. US Preventive Services Task Force., Curry Susan J., Krist Alex H., Owens Douglas K., Barry Michael J., Caughey Aaron B., Davidson Karina W., Doubeni Chyke A., Epling John W., Kemper Alex R., Kubik Martha., Landefeld C Seth., Mangione Carol M., Phipps Maureen G., Silverstein Michael., Simon Melissa A., Tseng Chien-Wen., Wong John B.(2018). Screening for Cervical Cancer: US Preventive Services Task Force Recommendation Statement. JAMA, 320(7), 674-686.

18. Wang Jiangrong., Elfström K Miriam., Andrae Bengt., Nordqvist Kleppe Sara., Ploner Alexander., Lei Jiayao., Dillner Joakim., Sundström Karin., Sparén Pär.(2020). Cervical cancer case-control audit: Results from routine evaluation of a nationwide cervical screening program. Int. J. Cancer, 146(5), 1230-1240.

19. Cheng Yanping., Yang Sheng., Shen Yang., Ding Bo., Wu Wenjuan., Zhang Yanqiu., Liang Geyu. (2020). The Role of High-Risk Human Papillomavirus-Related Long Non-Coding RNAs in the Prognosis of Cervical Squamous Cell Carcinoma. DNA Cell Biol., 39(4), 645-653.

20. Wang W P,An J S, Yao H W,Li N,Zhang Y Y,Ge L,Song Y,Wang M J,Yuan G W,Sun Y C,Huang M N,Wu L Y. (2019). Prevalence and attribution of high-risk HPV in different histological types of cervical cancer. Zhonghua fu chan ke za zhi, 54(5):293-300.

21. Zhang Shaokai. (2014). The association between HPV infection and cervical lesions and the natural history model of cervical cancer. Peking Union Medical College.

22. Stolnicu Simona., Barsan lulia., Hoang Lien., Patel Prusha., Terinte Cristina., Pesci Anna., Aviel-Ronen Sarit., Kiyokawa Takako., Alvarado-Cabrero Isabel., Pike Malcolm C., Oliva Esther., Park Kay J., Soslow Robert A.(2018). International Endocervical Adenocarcinoma Criteria and Classification 
(IECC): A New Pathogenetic Classification for Invasive Adenocarcinomas of the Endocervix. Am. J. Surg. Pathol., 42(2), 214-226.

23. Hodgson Anjelica., Olkhov-Mitsel Ekaterina., Howitt Brooke E., Nucci Marisa R., Parra-Herran Carlos. (2019). International Endocervical Adenocarcinoma Criteria and Classification (IECC): correlation with adverse clinicopathological features and patient outcome. J. Clin. Pathol., 72(5), 347-353.

24. Dong Hong, Li shuang, Wang Dan, Hu Ting, Lu Yunping, Wang Hui, Ma Ding, Wang Shixuan.(2010). Comparison of the Biological Behaviors and Prognostic Factors in Cervical Adenocarcinoma and Squamou Carcinoma. Acta Medicinae Universitatis Scientiae et Technologiae Huazhong, 39(02):254257.

25. White Kari., Garces Isabel C., Bandura Lisa., McGuire Allison A., Scarinci Isabel C.(2012). Design and evaluation of a theory-based, culturally relevant outreach model for breast and cervical cancer screening for Latina immigrants. Ethn Dis, 22(3), 274-80.

26. Mitchell H., Medley G., Gordon I., Giles G.(1995). Cervical cytology reported as negative and risk of adenocarcinoma of the cervix: no strong evidence of benefit. Br. J. Cancer, 71(4), 894-7.

27. Galic Vijaya., Herzog Thomas J., Lewin Sharyn N., Neugut Alfred I., Burke William M., Lu Yu-Shiang., Hershman Dawn L., Wright Jason D.(2012). Prognostic significance of adenocarcinoma histology in women with cervical cancer. Gynecol. Oncol., 125(2), 287-91.

28. Cheng Yifan, Xie Xing. Screening of cervical adenocarcinoma.(2016). Journal of practical obstetrics and gynecology, 32 (08) : 563-565.

29. Pirog E C., Kleter B., Olgac S., Bobkiewicz P., Lindeman J., Quint W G., Richart R M., Isacson C.(2000). Prevalence of human papillomavirus DNA in different histological subtypes of cervical adenocarcinoma. Am. J. Pathol., 157(4), 1055-62.

30. Hong J H., Tsai C S., Wang C C., Lai C H., Chen W C., Lee S P., Chang T C., Tseng C J.(2000). Comparison of clinical behaviors and responses to radiation between squamous cell carcinomas and adenocarcinomas/adenosquamous carcinomas of the cervix. Chang Gung Med J, 23(7), 396404.

31. Galic Vijaya., Herzog Thomas J., Lewin Sharyn N., Neugut Alfred I., Burke William M., Lu Yu-Shiang., Hershman Dawn L., Wright Jason D.(2012). Prognostic significance of adenocarcinoma histology in women with cervical cancer. Gynecol. Oncol., 125(2), 287-91.

\section{Figures}




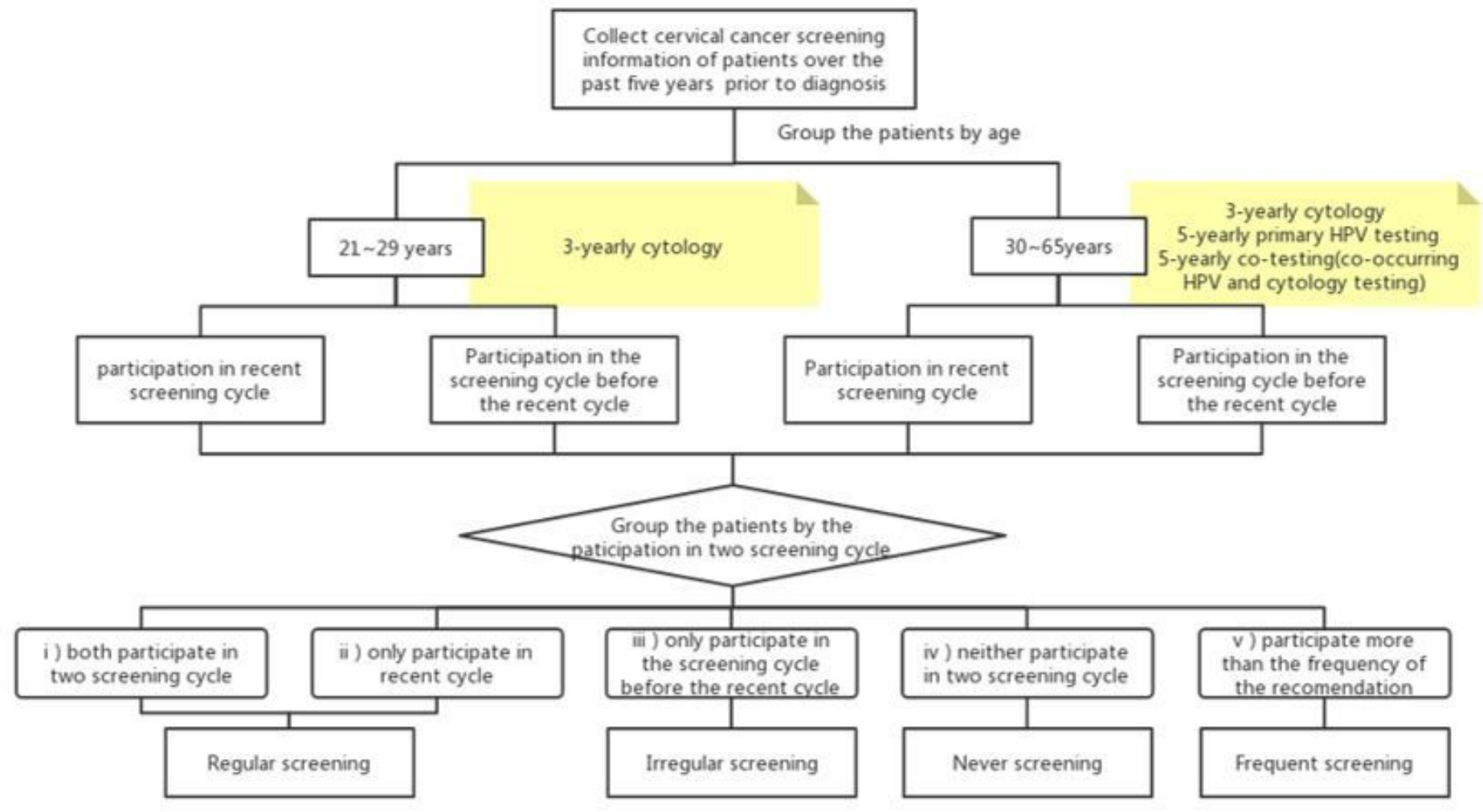

Figure 1

Grouping based on the participation in cervical screening. 


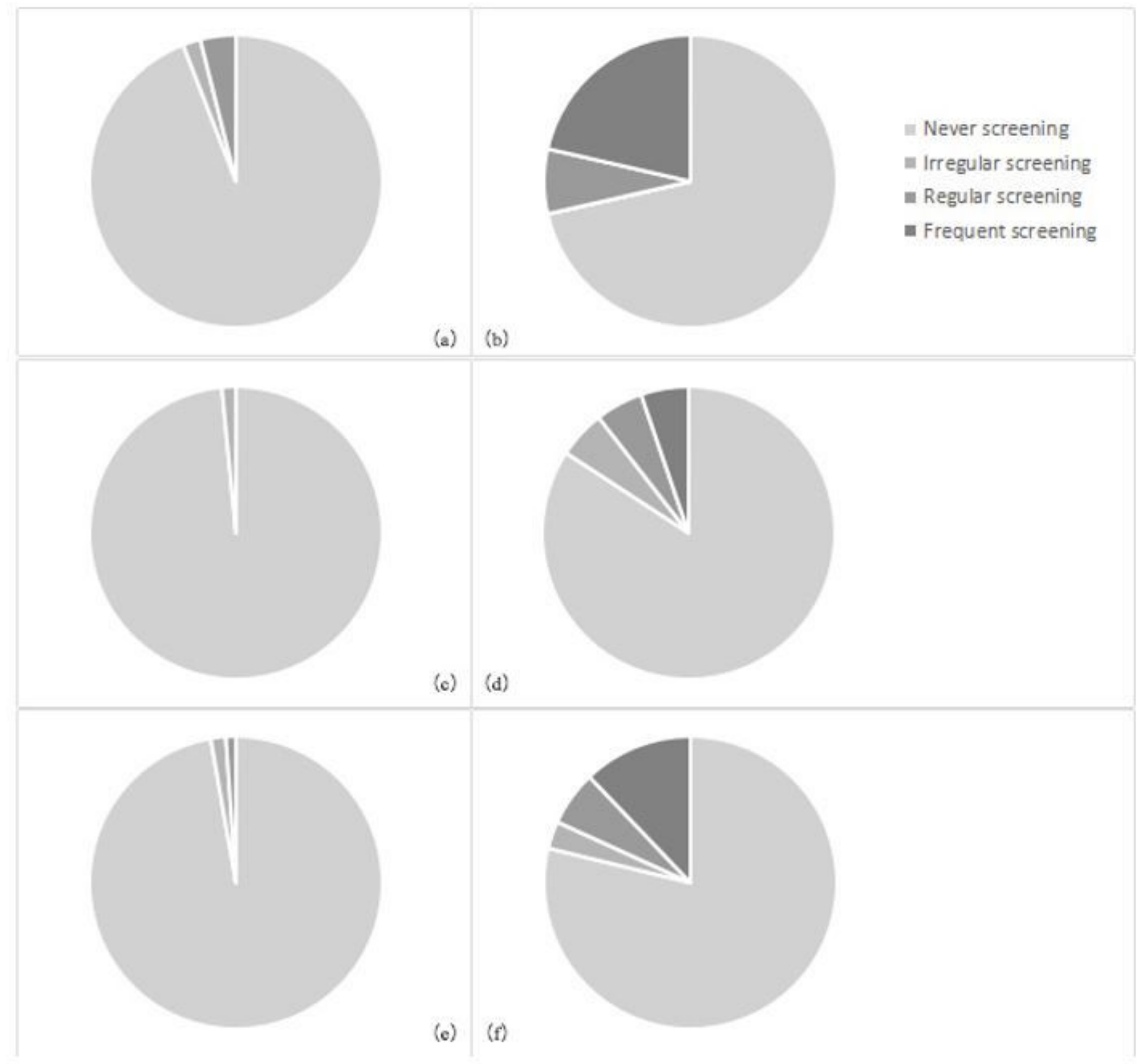

\section{Figure 2}

The characteristics of cervical screening participation among different histopathological types of cervical carcinoma in different cancer stage. i)Figure 3(a): Early-stage SCC: the percentages of never screening, irregular screening, regular screening and frequent screening are $94.1 \%, 2.0 \%, 3.9 \%, 0.0 \%$ respectively; (b)Early-stage AC: the percentages of never screening, irregular screening, regular screening and frequent screening are $71.4 \%, 0.0 \%, 7.1 \%, 21.4 \%$ respectively; (c)Advanced SCC: the percentages of never screening, irregular screening, regular screening and frequent screening are $98.5 \%, 1.5 \%, 0.0 \%, 0.0 \%$ respectively; (d)Advanced AC: the percentages of never screening, irregular screening, regular screening and frequent screening are $84.2 \%, 5.3 \%, 5.3 \%, 5.3 \%$ respectively; (e)SCC: the percentages of never 
screening, irregular screening, regular screening and frequent screening are $97.2 \%, 1.7 \%, 1.1 \%, 0.0 \%$ respectively; (f)AC: the percentages of never screening, irregular screening, regular screening and frequent screening are $78.8 \%, 3.0 \%, 6.1 \%, 12.1 \%$ respectively; ii)Cases without staging information and cases lost to follow-up were excluded. 